\title{
Wave packet revivals in a graphene quantum dot in a perpendicular magnetic field
}

\author{
J. J. Torres ${ }^{1,2}$ and E. Romera ${ }^{1,3}$ \\ ${ }^{1}$ Instituto Carlos I de Física Teórica y Computacional, \\ Universidad de Granada, Fuentenueva s/n, 18071 Granada, Spain \\ ${ }^{2}$ Departamento de Electromagnetismo y Física de la Materia, \\ Universidad de Granada, Fuentenueva s/n, 18071 Granada, Spain \\ ${ }^{3}$ Departamento de Física Atómica, Molecular y Nuclear, \\ Universidad de Granada, Fuentenueva s/n, 18071 Granada, Spain
}

(Dated: July 10, 2018)

\begin{abstract}
We study the time-evolution of localized wavepackets in graphene quantum dots in a perpendicular magnetic field, focusing on the quasiclassical and revival periodicities, for different values of the magnetic field intensities in a theoretical framework. We have considered contributions of the two inequivalent points in the Brillouin zone. The revival time has been found as an observable that shows the break valley degeneracy.

PACS numbers: 03.65.Pm, 73.63-b, 81.05.Uw
\end{abstract}

\section{INTRODUCTION}

Graphene, a single sheet of graphite, has attracted growing interest due to its remarkable and starling properties and its potential applications in nanoelectronics [1 5]. Many of these properties are attributed to the existence of a peculiar band structure constituted by quasifree electrons being described by a massless Dirac equation [6]. In fact, perfect tunneling of electron wave packets through potential barriers (the Klein paradox) [7] or the unconventional quantum Hall effect, have been experimentally confirmed in graphene [8]. It has been also argued that graphene in a magnetic field is a promising system for the experimental observation of the highly oscillatory motion of the electric current known as zitterbewegung [9-12]. In addition, revivals of electric currents in graphene in the presence of a magnetic field [13] have been theoretically predicted and an exhaustive study of quantum wave-packet revivals, fractional revivals, classical periodicity in graphene was reported [13, 14].

In recent times, graphene quantum dots have been widely studied, both theoretically and experimentally. It has been reported that there are different theoretical approaches which allow to electrostatically define a graphene quantum dot, or to create it by cutting flakes of graphene [15 22]. Also, there has been an analysis of the level statistics of the graphene quantum dots observed in experiments (for quantum dots smaller than $100 \mathrm{~nm}$ ) which has concluded that it is well described by the theory of chaotic Dirac neutrino billiards [23]. On the other hand, new experiments have been designed for the creation of graphene quantum dots 24 26] by etching or scratching graphene islands including the study of energy levels in magnetic fields [26, 27]. Additionally, graphene quantum dots have been point out to be very attractive as spin qubits in quantum information processing [28].

The temporal evolution of wavepackets, relativistic and nonrelativistic, displays interesting revival phenomena due to quantum interference. Several types of periodicity may emerge depending on the energy eigenvalue spec- trum, in this regard revivals and fractional revivals have raised great interest during recent years [29]. Propagating wave packets initially evolve quasiclassically and oscillate with a period $T_{c l}$, and later the wavepackets spread and collapse. For longer times, the wavepackets evolve in the so-called revival time $T_{r e v}$ to a state that closely reproduces its initial waveform. Fractional revivals appear as a temporal self-splitting of the initial wavepacket into a collection of a number of scaled copies at times $t=p T_{\text {rev }} / q$, with $p$ and $q$ mutually prime. Assuming that the initial wavepacket is a superposition of the eigenstates $u_{n}$ sharply peaked around some $n_{0}$, revival times can be obtained from the Taylor series of the energy spectrum $E_{n}$ around $E_{n_{0}}$ as $T_{\text {rev }}=4 \pi \hbar /\left|E_{n_{o}}^{\prime \prime}\right|$. Revivals have been investigated theoretically and experimentally in nonlinear quantum systems 29 34]. Recently, revivals of electric current in monolayer graphene in the presence of an external magnetic field have been also described [13, 14].

This article is organized as follows. In section II the phenomenon of wavepacket revivals in a graphene quantum dot in a magnetic field using a model Hamiltonian is studied. We discuss on the relevance of quantum revivals for the characterization of the breaking of the valley degeneracy in graphene quantum dots.

\section{WAVE PACKET REVIVALS FOR GRAPHENE QUANTUM DOTS IN A MAGNETIC FIELD}

To study wave packet revivals in a graphene quantum dot in a perpendicular magnetic field $\mathbf{B}$ we consider the model Hamiltonian for electrons in the valley-isotropic form which is given by [21]

$$
H_{\tau}=v_{F}(\mathbf{p}+e \mathbf{A}) \cdot \boldsymbol{\sigma}+\tau V(r) \sigma_{z} .
$$

We use the symmetric gauge for the vector potential, $\mathbf{A}=B / 2(-y, x, 0)=B / 2(-r \sin \phi, r \cos \phi, 0)$, where $\phi$ the polar angle, $v_{F}=10^{6} \mathrm{~m} / \mathrm{s}$ the Fermi velocity, and $\tau= \pm 1$ differentiates the two valleys $K_{1}$ and $K_{2} . \sigma$ are 

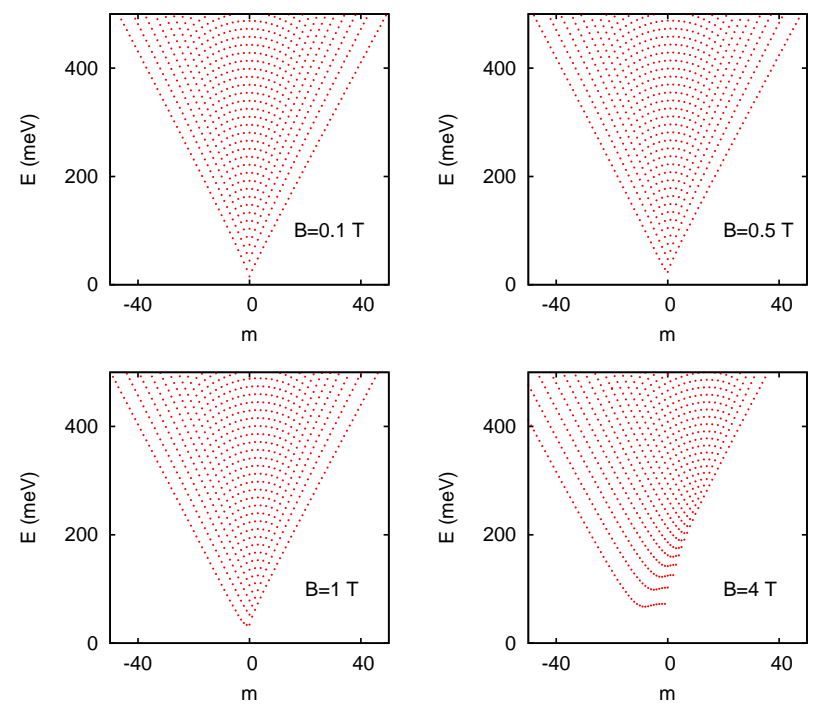

FIG. 1. Positive energy spectrum for a graphene Quantum Dot, with $R=70 \mathrm{~nm}$, versus the quantum number $m$ and different values of the magnetic field $B$ ( $K_{1}$ point).

\begin{tabular}{|l|ll|ll|}
\hline \hline$B(T)$ & $T_{c l}(\mathrm{ps})$ & $T_{r e v}(\mathrm{~ns})$ & $T_{c l}^{\prime}(\mathrm{ps})$ & $T_{r e v}^{\prime}(\mathrm{ns})$ \\
\hline 0.1 & 0.14 & 115.8 & 0.14 & 120.7 \\
0.5 & 0.14 & 6.8 & 0.14 & 7.6 \\
1 & 0.14 & 2.3 & 0.14 & 2.6 \\
4 & 0.14 & 0.2 & 0.14 & 0.3 \\
\hline \hline
\end{tabular}

TABLE I. Classical period $T_{c l}$ and revival time $T_{r e v}$ in the two valleys $\left(K_{1}\right.$ and $\left.K_{2}\right)$ in the band structure of graphene quantum dot for different values of the magnetic field $B$ and for an initial wave packet with $n_{0}=20, m=0, \sigma=1.1$.

Pauli's spin matrices in the basis of the two sublattices of $\mathrm{A}$ and $\mathrm{B}$ atoms. We assume the confinement potential in [21], which is a mass-related potential energy $V(r)$ coupled to the Hamiltonian via the $\sigma_{z}$ Pauli matrix, where $V(r)=0$ for $r<R$ and $V(r)=\infty$ for $r>R$, that is, tends to infinity in the edge of the dot.

Upon introducing the magnetic length $l_{B}=\sqrt{\hbar /(e B)}$ and using the fact that $H_{\tau}$ commutes with the total angular momentum operator $J_{z}=L_{z}+\frac{\hbar}{2} \sigma_{z},\left[H_{\tau}, J_{z}\right]=0$, the solution of the Dirac equation $H_{\tau} \psi(r, \phi)=E \psi(r, \phi)$ (where $\psi(r, \phi)=\left[\psi_{1}(r, \phi), \psi_{2}(r, \phi)\right]$ is a two-component spinor) is given by [21]:

$$
\begin{aligned}
& \psi_{1}(r, \phi)=c e^{i m \phi} r^{m} e^{-r^{2} / 4 l_{B}^{2}} L\left(\frac{k^{2} l_{B}^{2}}{2}-(m+1), m, r^{2} / 2 l_{B}^{2}\right) \\
& \psi_{2}(r, \phi)=c e^{i(m+1) \phi} r^{m} e^{-r^{2} / 4 l_{B}^{2}}
\end{aligned}
$$$$
\times \frac{r / l_{B}}{k l_{B}} L\left(\frac{k^{2} l_{B}^{2}}{2}-(m+1), m+1, r^{2} / 2 l_{B}^{2}\right)
$$

where $L(a, b, z)$ is the generalized Laguerre function and $c$ is a normalization constant. The characteristic equation for the allowed eigenenergies $E$ of the Quantum Dot using the boundary condition for a circular confinement [21, 35] $\psi_{2} / \psi_{1}=\tau \exp [i \phi]$ has been obtained in [21] and it can be written as

$$
\begin{aligned}
& \left(1-\tau \frac{k l_{B}}{R / l_{B}}\right) L\left(\frac{k^{2} l_{B}^{2}}{2}-(m+1), m, R^{2} / 2 l_{B}^{2}\right) \\
& +L\left(\frac{k^{2} l_{B}^{2}}{2}-(m+2), m+1, R^{2} / 2 l_{B}^{2}\right)=0 .
\end{aligned}
$$

(a)

(b)
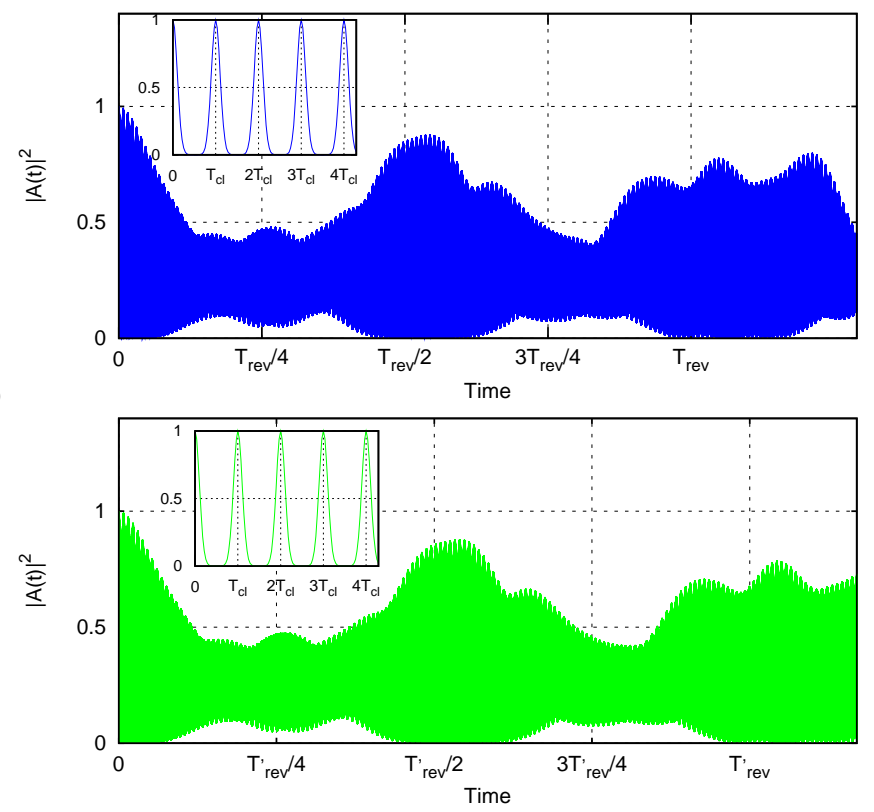

FIG. 2. Time dependence of $|A(t)|^{2}$ for an initial spherically symmetric Gaussian wavepacket with $n_{0}=20, \sigma=1.1, B=$ $0.2 \mathrm{~T}$ and $R=70 \mathrm{~nm}$ for (a) the valley $K_{1}\left(T_{\text {rev }}=28 \mathrm{~ns}\right)$ and (b) the valley $K_{2}\left(T_{\text {rev }}^{\prime}=31 \mathrm{~ns}\right)$.

The spectrum of electrons confined in a circular graphene quantum dot in a perpendicular magnetic field is given by $E(n, m, \tau)$ verifying (2) and (3) and the energy eigenfunctions are $\psi_{(n, m)}(r, \phi)$. Schnez et al. [21] have analyzed the limits for $B=0$ and $R / l_{B} \rightarrow \infty$ cases. For $B=0$ the eigenenergies are given by the characteristic equation $\tau J_{m}(k R)=J_{m+1}(k R)$. On the other hand, they showed that the energy levels converge to the landau levels $E_{m}=\hbar v_{F} k_{m}= \pm v_{F} \sqrt{2 e \hbar B(m+1)}$ for $R / l_{B} \rightarrow \infty$

The initial wave packets will be constructed as a superposition of eigenstates assuming a Gaussian population of energy levels

$$
\Psi(r, \phi)=\sum_{n, m} c_{n, m} \psi_{(n, m)}(r, \phi)
$$

that is, we choose $c_{n, m}$ Gaussianly distributed and centered around some $n_{0}, m_{0}$ with width $\sigma$ (See for instance 
Refs. 32, 36, 37]). We will study wave packet dynamic of this initial wave packet for different values of the intensity of the magnetic field $B$. The particular assumption we have made to construct the initial wavepacket has an influence in the results (see i. e. 32]). Moreover, the construction of a realistic initial wavepacket (i.e. by laser excitation) would require further considerations.

We start studying the special case of a radially symmetric wave packet, that is, a superposition of purely $m=0$ states. For an initial wave packet sharply peaked around a central $n_{0}$, the different time scales can then be identified from the coefficients of the Taylor expansion of the energy spectrum $E_{n}$ around $E_{n_{0}}$ :

$$
E_{n}=E_{n_{0}}+\frac{2 \pi \hbar}{T_{c l}}\left(n-n_{0}\right)+\frac{4 \pi \hbar}{T_{r e v}}\left(n-n_{0}\right)^{2}+\ldots
$$

In Table I (middle column) the times scales $T_{c l}$ and $T_{r e v}$ have been numerically fitted from energies of Fig. 1. The quantum number dependence of the energy eigenvalues is necessary to determine the classical period and the revival time in a system. In Fig. 1 we plot the $n$ and $m$ dependence of the energies $E_{n, m}$ for $B=0.1,0.5,1$ and $4 \mathrm{~T}$ and for $R=70 \mathrm{~nm}$ in the valley $K_{1}$.

To analyze the evolution of the wave packet we shall use the autocorrelation function

$$
A(t)=\int r d r d \phi \psi^{*}(r, \phi, 0) \psi(r, \phi, t) .
$$

An alternative approach based on information entropies has been recently proposed 38]. The occurrence of revivals correspond to the approximate return of $|A(t)|^{2}$ to its initial value of unity. The time evolution of the autocorrelation function for an initial wave packet with $n_{0}=20, m=0 \sigma=1.1, R=70 \mathrm{~nm}$ and $B=0.2 \mathrm{~T}$ is showed in Fig. 2 (top panel). We can see that at early times the Gaussian wave packet evolves quasiclassically in the inset of the figures. For longer time scales the wave packet initially spreads and delocalizes. A revival of the wavepacket can be observed for $T_{\text {rev }} / 2$ (mirror revival [32]).

Let us turn to examine quantum revivals in the two inequivalent points in the Brillouin zone $K_{1}$ and $K_{2}$ which corresponds with Hamiltonian (11) with $\tau=1$ and $\tau=-1$, respectively. We have calculated the classical and revival times for different values of the magnetic field $B$ which are summarized in table II. The quasiclassical evolution is analogous in both valleys as we can see in the insets of Fig. 2. We have found both theoretically and by simple inspection of the behavior of the autocorrelation function in Fig. 2, that $T_{\text {rev }}$ differs in both zones clearly for a magnetic field $B=0.2 T$. In fact, it is known that the valley degeneracy is broken by the perpendicular magnetic field [20, 28]. So, we have found the revival time as an observable which shows explicitly the breaking of valley degeneracy in a graphene quantum dots. We would like to point out that the breaking of the valley degeneracy is a prerequisite for spin-based quantum computing in graphene quantum dots. As the
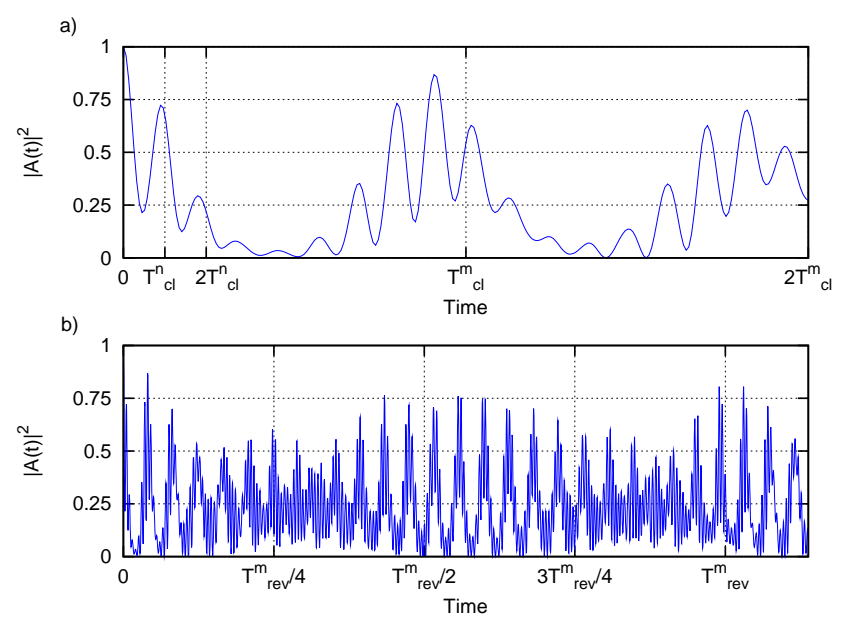

FIG. 3. Time dependence of $|A(t)|^{2}$ for initial wavepackets with $B=4 \mathrm{~T}, R=70 \mathrm{~nm}, n_{0}=1, m_{0}=-15$ and $\sigma_{n}=\sigma_{m}=$ 0.7 in the valley $K_{1}$.(a) First classical periods of motion with $T_{c l}^{(n)}=0.08 \mathrm{ps}, T_{c l s}^{(m)}=0.65 \mathrm{ps}$ (b) Long time dependence with $T_{\text {rev }}^{(n)}=2.09 \mathrm{ps}$ and $T_{\text {rev }}^{(m)}=14.50 \mathrm{ps}$.

magnetic field is growing the revivals times are equalizing as we expect for Landau levels regime [13].

Finally, we consider an initial superposition state with coefficients taken from a Gaussian distribution centered around $m=-15$ and $n=1$ for $B=4 T$. In this case the energy levels are characterized by two quantum numbers $E(n, m)$. Systems with two quantum numbers offer richer possibilities for wave packet revivals (for a theoretical analysis see [32]). The classical periods yield straightforwardly $T_{c l}^{n}=0.078 \mathrm{ps}$ and $T_{c l}^{m}=0.65 \mathrm{ps}$ (from [32] $T_{c l}^{(n)}=2 \pi \hbar /\left|\frac{\partial E}{\partial n}\right|_{n_{0}, m_{0}}$ and $T_{c l}^{(m)}=2 \pi \hbar /\left|\frac{\partial E}{\partial m}\right|_{n_{0}, m_{0}}$, respectively). Now, the long-time revival structure depends on three possible times, given by [32] $T_{\text {rev }}^{(n)}=$ $4 \pi \hbar /\left|\frac{\partial^{2} E(n, m)}{\partial n^{2}}\right|_{n_{0}, m_{0}}, T_{r e v}^{(m)}=4 \pi \hbar /\left|\frac{\partial^{2} E(n, m)}{\partial m^{2}}\right|_{n_{0}, m_{0}}$ and $T_{r e v}^{(n, m)}=2 \pi \hbar /\left|\frac{\partial^{2} E(n, m)}{\partial n \partial m}\right|_{n_{0}, m_{0}}$, and the revival structure is constructed on the interplay between these times. This is shown in Fig. 3 for $B=4 T$. In the top panel it is apparent that at short times the quasiclassical oscillatory behavior with period $T_{c l}^{(n)}=0.078 \mathrm{ps}$. At medium times the quasiclassical behavior corresponding to $T_{c l}^{(m)}=0.65 \mathrm{ps}$ is superimposed on the first oscillations. In the bottom panel revivals can be seen at $t \approx 8.5 p s$ as an interplay between the revival times, as we would expect. Snapshots of the numerical simulation of the probability density are given in Fig. [4 at several times. It is apparent that the regeneration of the density function is given at time $t=8.65 \mathrm{ps}$.

We want to remark that the typical scattering times should be taken into account for a more realistic description of this problem. Moreover, it would be interesting 

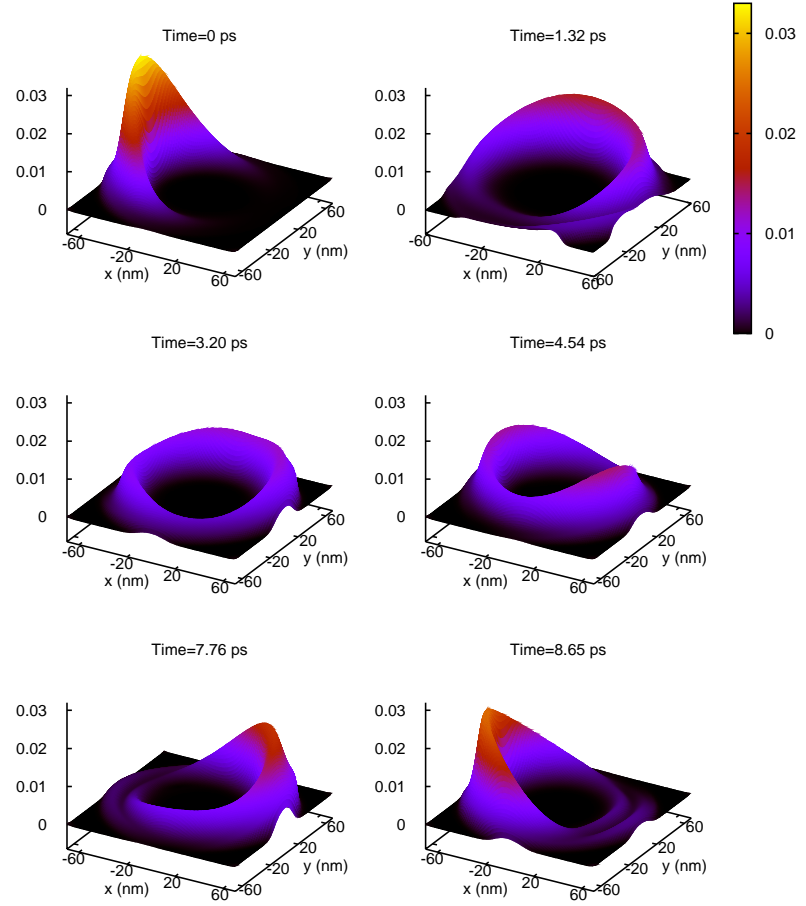

FIG. 4. Snapshots of the probability density $|\psi(x, y)|^{2}$ for an initial gaussian wavepacket with $n_{0}=1, m_{0}=-15, \sigma_{n}=$ $\sigma_{m}=0.7, B=4 T$ and $R=70 \mathrm{~nm}$. It is noticeable the existence of fractional revivals after the delocalization of the initial wave packet (middle panels) and the revival at $t=$ $8.65 \mathrm{ps}$. to understand the influence of shape deformations, impurities, or interactions on revivals, so a more detailed study of this topic could be made in a analogous way that has been done before (see i.e. [13, 14]) in graphene in a perpendicular magnetic field.

\section{CONCLUSIONS}

In this work we have presented time-dependent effects in the propagation of wavepackets on graphene quantum dots for different values of the magnetic field. When the wavepacket is sufficiently localized around some central quantum numbers $\left(n_{0}, m_{0}\right)$, it evolves quasiclassically. At later times, it spreads leading to the collapse of the classical oscillations, but at times that are multiple or rational fractions of $T_{r e v}$, the wavepacket regains its initial form. We have also shown revival time as an observable sign of breaking the valley degeneracy, that it is known as a prerequisite for spin-based quantum computing. It can be underline that we have found revivals of the electron wavepacket in a Dirac system and we suggest that this realistic system could be used to simulate relativistic quantum revivals [13, 39].

\section{ACKNOWLEDGMENTS}

We want to thank J. A. López-Villanueva and F. de los Santos for fruitful discussions. This work was supported by projects FQM-165/0207, FIS2008-01143, FQM-01505 and FIS2009-08451.
[1] A. H. Castro Neto, F. Guinea, N. M. R. Peres, K. S. Novoselov and A. K. Geim, Rev. Mod. Phys. 81, 109$162(2009)$

[2] A. K. Geim, Science 324, 1530 (2009).

[3] K. S. Novoselov Phys. World 22(8), 27 (2009)

[4] C. Lee, X. Wei, J.W. Kysar, and J. Hone, Science 321, 385 (2008); A.A. Balandin et al., Nano Lett. 8, 902 (2008).

[5] J.H. Chen, C. Jang, S. Xiao, M. Ishigami, and M.S. Fuhrer Nature Nanotech. 3, 206 (2008).

[6] K.S. Novoselov et al., Nature 438, 197 (2005).

[7] M.I. Katsnelson, K.S. Novoselov and A.K. Geim, Nature Physics 2, 620 (2006), A.F. Young and P. Kim, Nature Phys. 5, 222 (2009); N. Stander, B. Huard and D. Goldhaber-Gordon, Phys. Rev. Lett. 102, 026807 (2009).

[8] A.K. Geim and K.S. Novoselov, Nat. Mater. 6, 183 (2007).

[9] T.M. Rusin and W. Zawadzki, Phys. Rev. B 78, 125419 (2008).

[10] T.M. Rusin and W. Zawadzki, Phys. Rev. B 76, 195439 (2007).

[11] T.M. Rusin and W. Zawadzki, Phys. Rev. B 80, 045416 (2009).

[12] G. M. Maksimova, V. Ya. Demikhovskii, and E. V. Frolova, Phys. Rev. B 78, 235321 (2008)
[13] E. Romera and F. de los Santos, Phys. Rev. B 80, 165416 (2009).

[14] V.Krueckl and T. Kramer, New J. Phys. 11, 093010 (2009).

[15] P. G. Silvestrov and K. B. Efetov, Phys. Rev. Lett. 98 016802 (2007).

[16] H. Y. Chen, V. Apalkov, and T. Chakraborty, Phys. Rev. Lett. 98, 186803 (2007).

[17] A. Matulis and F. M. MPeeters, Phys. Rev. B 77, 115423 (2008).

[18] J. H. Bardarson, M. Titov and P. W. Brouwer, Phys. Rev. Lett. 102, 226803, (2009)

[19] B. Trauzettel, D. V. Vulaev, D. Loss, and G. Burkard, Nature Phys. 3, 192 (2007).

[20] P. Recher, J. Nilsson, G. Burkard, and B. Trauzettel, Phys. Rev. B 79, 085407 (2009).

[21] S. Schnez, K. Ensslin, M. Sigrist, and T. Ihn, Phys. Rev. B 78, 195427 (2008).

[22] A. De Martino, L. DellAnna, and R. Egger, Phys. Rev. Lett. 98, 066802 (2007).

[23] Ponomarenko, F. Schedin, M. I. Katsnelson, R. Yang, E. W. Hiel, K. S. Novoselov, A. K. Geim, Science 320, 356 (2008).

[24] C. Stampfer, J. Güttinger, F. Molitor, D. Graf, T. Ihn, and K. Ensslin, Appl. Phys. Lett. 92, 012102 (2008). 
[25] L. Ponomarenko, F. Schedin, M. Katsnelson, R. Yang, E. Hill, K. Novoselov and A. Geim, Science 320, 356 (2008).

[26] S. Schnez, F. Molitor, C. Tampfer, J. Güttinger, I. Shorubalko, T. Ihn, and K. Ensslin, Appl. Phys. Lett. 94, 012107 (2009).

[27] J. Güttinger, C. Stampfer, T. Frey, T. Ihn, and K. Ensslin, Phys. Status Solidi B 246, 2553 (2009).

[28] P. Recher and B. Trauzettel, Quamtum dots and spain qubits in graphene arXiv:1004.2136 (2010).

[29] J.H. Eberly, N.B. Narozhny, and J.J. SánchezMondragón, Phys. Rev. Lett. 44, 1323 (1980).

[30] I.Sh . Averbukh and J.F. Perelman, Phys. Lett. A 139, 449 (1989); Acta Phys. Pol. A78, 33 (1990).

[31] D.L. Aronstein and C.R. Stroud Jr., Phys. Rev. A 55, 4526 (1997).

[32] R.W. Robinett, Phys. Rep. 392, 1 (2004).
[33] J.A. Yeazell, M. Mallalieu, and C.R. Stroud, Jr., Phys. Rev. Lett. 64, 2007 (1990).

[34] G. Rempe, H. Walther, and N. Klein, Phys. Rev. Lett. 58, 353 (1987); T. Baumert et al., Chem. Phys. Lett. 191, 639 (1992); M.J.J. Vrakking, D.M. Villeneuve, and A. Stolow, Phys. Rev. A 54, R37-R40 (1996); A. Rudenko et al., Chem. Phys. 329, 193 (2006).

[35] M. V. Berry and R. J. Mondragon, Proc. R. Soc. London, Ser. A 412, 53 (1987).

[36] J. Banerji and S. Ghosh, J. Phys. B 39, 1113 (2006).

[37] R. Bluhm, V. A. Kostelecky and J. A. Porter, Am. J. Phys. 64, 944 (1996).

[38] E. Romera, and F. de los Santos, Phys. Rev. Lett. 99,263601 82007); Phys. Rev. A 78, 013837 (2008).

[39] P. Strange, Phys. Rev. Lett. 104, 120403 (2010). 